\title{
Fundamental Limitations of Power Control in WCDMA
}

\author{
Fredrik Gunnarsson \\ Division of Communication Systems \\ Department of Electrical Engineering \\ Linköpings universitet, SE-581 83 Linköping, Sweden \\ WWW: http://Www. comsys.isy.liu.se \\ Email: fred@isy.liu.se \\ 4th December 2001

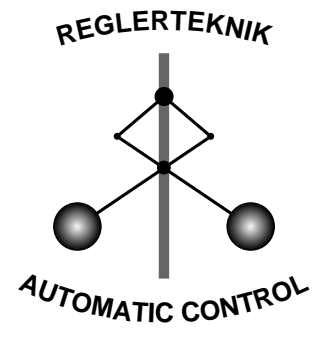 \\ LINKÖPING
}

Report No.: LiTH-ISY-R-2404

Submitted to VTC'01 Fall, Atlantic City, NJ, USA

Technical reports from the Communication Systems group in Linköping are available at http://www.control.isy.liu.se/publications. 


\begin{abstract}
Power control is considered as an important means to combat near-far fading effects and maintain acceptable connections in wireless communications systems. When applying power control in practice, the performance is restricted by a number of fundamental limitations. Here, these are addressed from a control theory perspective. Limited update rate, limited feedback bandwidth, time delays, measurement errors, feedback errors, and filtering effects among other aspects all affect the resulting performance, and are related to radio channnel characteristics. Simulations further illustrate the hampering effects.
\end{abstract}

Keywords: power control, WCDMA, controller bandwidth, time delay, stability, filtering 


\title{
Fundamental Limitations of Power Control in WCDMA
}

\author{
Fredrik Gunnarsson, IEEE Member \\ Division of Control and Communications \\ Department of Electrical Engineering \\ SE-581 83 LINKÖPING, SWEDEN \\ Email: freddisy.liu.se
}

\begin{abstract}
Power control is considered as an important means to combat near-far fading effects and maintain acceptable connections in wireless communications systems. When applying power control in practice, the performance is restricted by a number of fundamental limitations. Here, these are addressed from a control theory perspective. Limited update rate, limited feedback bandwidth, time delays, measurement errors, feedback errors, and filtering effects among other aspects all affect the resulting performance, and are related to radio channnel characteristics. Simulations further illustrate the hampering effects.
\end{abstract}

\section{INTRODUCTION}

While the demand for access to services in wireless communications systems is exponentially growing, an increased interest in utilizing the available resources efficiently can be observed. A consequence of the limited availability of radio resources is that the users have to share these resources. Power control is seen as an important means to reduce mutual interference between the users, while compensating for timevarying propagation conditions. As with any feedback control system, some fundamental limitations do come into play. The objective with this paper is to express these effects using a control theory framework.

Power control has been an area subject to extensive research in recent years. Some surveys of previous work include [1], [2], [3].

If full information of the propagation conditions between mobiles and base stations are known, the transmitter powers of every transmitter could be computed in a centralized fashion. One approach is to aim at the same SIR at every receiver (SIR balancing) suitable for single service systems (see e.g., [4]). The radio network itself puts some overall restrictions on the tractability of the transmitter power control algorithms. If there exists transmitter powers to meet the individual requirements of the users, the power control problem is said to be feasible [5], [6].

To actually implement a centralized power control solution is not plausible in practice due to the signaling overhead. Instead, such schemes serve as performance bounds, to implementationally appealing distributed solutions. These include the Distributed Power Control (DPC) algorithm [7], which converge to the centralized solution if the power control problem is feasible. Other important decentralized proposals include [8], [9], [10], [11], [12], [13], [14] aiming at different perspectives of power control, such as constrained power levels, fixed-step power updates, measurement related issues,

This is the article version of the talk given at the 6th Swedish CDMA Workshop, Dec. 2000. The work is supported by the competence center ISIS Linköpings universitet, and in cooperation with Ericsson Research, which all are acknowledged. time delays and problems when the power control problem is infeasible.

The system model and the notation is introduced in Section 2, together with a discussion on ideal distributed power control and control theory modelling aspects. Standardized power control algorithms for WCDMA are discussed in Section 3. Various fundamental limitations and illuminating simulations are in focus in Section 4. Section 5 provides some concluding remarks.

\section{SySTEM MODEL}

To emphasize that the discussion applies to both the up- and downlink, we consider a system of $m$ transmitters and $m$ receivers. In an uplink situation, the transmitters and the active mobile stations are equivalent, while the base stations are seen as equipped with a number of receivers - one per connected mobile station, and vice versa in the downlink. Thereby, there is a one-to-one correspondence between transmitters and the connected receivers. The base station assignments are assumed fixed over the time frame of the analysis, which is natural, since updates are much more infrequent than power level updates.

\section{A. Notation}

Most quantities in this paper can be expressed using either logarithmic (e.g. $d B$ or $d B m$ ) or linear scale. To avoid confusion we will employ the convention of indicating linearly scaled values with a bar. Thus $\bar{g}_{i j}(t)$ is a value in linear scale and $g_{i j}(t)$ the corresponding value in logarithmic scale.

Assume that the $m$ transmitters are transmitting using the powers $p_{i}(t), i=1, \ldots, m$. The signal between transmitter $i$ and receiver $j$ is attenuated by the power gain $g_{i j}(t)(<0)$. Thus the corresponding connected receiver will experience a desired signal power $p_{i}(t)+g_{i i}(t)$ and an interference from other connections plus noise $I_{i}(t)$. The signal-to-interference ratio (SIR) at receiver $i$ can be defined by

$$
\gamma_{i}(t)=p_{i}(t)+g_{i i}(t)-I_{i}(t)
$$

The focus is on a specific connection, and interference is therefore considered as an independent disturbance. This is not true in practice, but it suits our modeling purposes.

We will only discuss the Quality of Service (QoS) in terms of SIR. The individual quality objectives at each receiver $i$ are assumed expressed as target SIR:s $\gamma_{i}(t)$, possibly reconsidered regularly by outer control loops. The outer loop update rate is typically orders of magnitude slower, and the target SIR:s will therefore be considered constant. 


\section{B. Channel Characteristics}

A Vehicular A channel model will be utilized throughout the paper. Power gain values over $20 \mathrm{~m}$ are depicted in Fig. $1 \mathrm{a}$. The frequency content of the power gain can be described independent of mobile velocity by expressing it with respect to the spatial frequency $\xi\left[\mathrm{m}^{-1}\right]$. As seen in Fig. $1 \mathrm{~b}$, most of the frequency content is concentrated below $80 \mathrm{~m}^{-1}$. For example the velocity $v \mathrm{~m} / \mathrm{s}$ means that the disturbance energy is concentrated below $80 v \mathrm{~Hz}$.
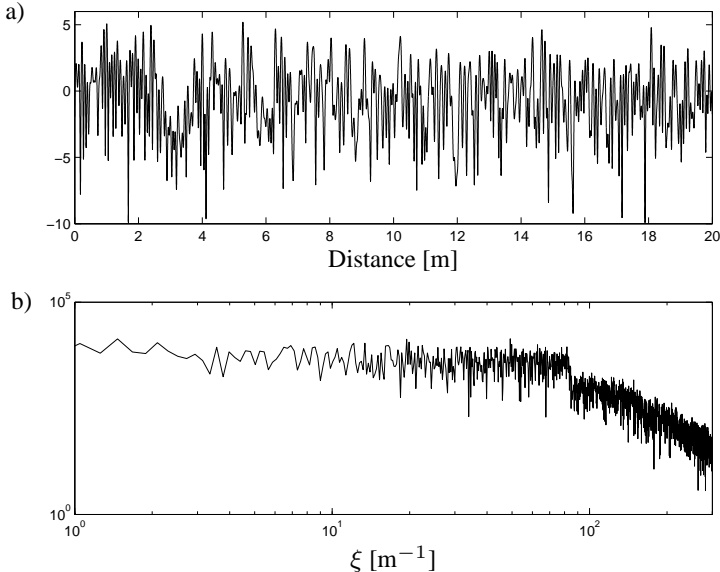

Fig. 1. Realization of Vehicular A power gain a) with respect to travelled distance and $b$ ) in the frequency domain w.r.t. spatial frequency.

\section{Distributed Power Control Algorithms}

The distributed power control algorithms are based on local feedback information, typically related to SIR. An integrating control algorithms is foundational:

$$
p_{i}(t+1)=p_{i}(t)+\beta+\left(\gamma_{i}^{t}(t)-\gamma_{i}(t)\right)=p_{i}(t)+\beta e_{i}(t),
$$

Essentially, the control error $e_{i}(t)$ is fed back from the receiver to the transmitter where it is integrated. Yet simple, this relates to most of the proposed algorithms to date. For example, the Distributed Power Control (DPC) algorithm is obtained with $\beta=1$. Note that the algorithm contains a processing delay of one update interval. Additional delays of $n$ update intervals are present in practice, and can be modeled as delayed power updates:

$$
\gamma_{i}(t)=p_{i}(t-n)+g_{i i}(t)-I_{i}(t) .
$$

Furthermore, the SIR measurements are subject to noise, modeled as additive and Gaussian

$$
\hat{e}_{i}(t)=\gamma_{i}^{t}(t)-\gamma_{i}(t)-w_{i}(t)=e_{i}(t)-w_{i}(t) .
$$

The actual time between consecutive power updates, the sample interval $T_{s}$, varies from systems to system. For example $T_{s}=0.48 \mathrm{~s}$ in GSM and $T_{s}=1 / 1500 \mathrm{~s}$ in WCDMA. To avoid confusion, we let the time index $t$ represent instants of power level updates in the transmitters. Seemingly, this notation is equal to the assumption of synchronous updates, but the only needed assumption is that all transmitters update their power levels within the time frame of one sample interval.

\section{Power Control from a Control Theory Perspective}

The local dynamical behaviour can be conveniently described using a control theory framework. Introduce the timeshift operator $q$ as

$$
q^{-n} p(t)=p(t-n), q^{n} p(t)=p(t+n)
$$

For a more rigid discussion on a $q$-operator algebra, the reader is referred to [15]. The intuitive relations to the complex variable $z$ of the $z$-transform are also addressed.

The integrator control algorithm in (2) can be rewritten using the time-shift operator

$$
p_{i}(t)=\frac{\beta}{q-1} \hat{e}_{i}(t)=R(q) \hat{e}_{i}(t)
$$

When subject to time delays and measurement errors, the distributed power control loop (or local loop) can be depicted as in Fig. 2

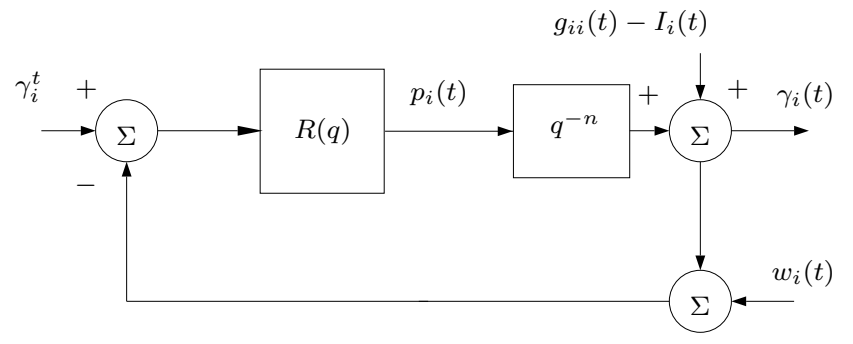

Fig. 2. The local loop dynamics when employing the general linear control algorithm $R(q)$. The measurements are subject to additive noise $w_{i}(t)$, and the output powersare delayed by $n$ samples.

Clearly, the power control objective is to maintain $\gamma_{i}(t)=$ $\gamma_{i}^{t}(t)$ or equivalently $e_{i}(t)=0$. From the block diagram in Fig. 2, we obtain

$$
\begin{aligned}
& \gamma_{i}(t)=G(q) \gamma_{i}^{t}(t)+S(q)\left(g_{i i}(t)-I_{i}(t)\right)+G(q) w_{i}(t) \\
& e_{i}(t)=S(q)\left(\gamma_{i}^{t}(t)-g_{i i}(t)+I_{i}(t)\right)+G(q) w_{i}(t),
\end{aligned}
$$

where the dynamics is described by

$$
G(q)=\frac{\beta}{q^{n}(q-1)+\beta}, S(q)=\frac{q^{n}(q-1)}{q^{n}(q-1)+\beta}
$$

$G(q)$ is referred to as the closed-loop system, and $S(q)$ as the sensitivity function. Note that $S(q)=1-G(q)$. The corresponding relations in the frequency domain are obtained by replacing $q$ by $e^{i \omega T_{s}}$. In practice, this will be estimated from simulated (or measured) data using the emprircal transfer function estimate (ETFE) [16]

$$
\hat{S}\left(e^{i \omega}\right)=\frac{\hat{E}_{i}\left(e^{i \omega}\right)}{\hat{G}_{i i}\left(e^{i \omega}\right)},
$$

where $E_{i}\left(e^{i \omega}\right)$ and $G_{i i}\left(e^{i \omega}\right)$ are estimates of the fourier transform of the signals $e_{i}(t)$ and $g_{i i}(t)$ (for example using smoothed FFT:s). 
The closed-loop system describes the tracking capability of the control algorithm, while the sensitivity function relates to the disturbance suppression performance. The effects of measurement (sensor) errors, however, is also captured by the closed-loop system. Furthermore, local loop stability is related to properties of $G(q)$ [17]. A fundamental constraint on the linear control performance and error suppression can be expressed in terms of the Bode integral constraint on $S$.

$$
\int_{0}^{\pi} \log \left|S\left(e^{i \omega}\right)\right| d \omega=0
$$

This means that it is not possible to obtain $S\left(e^{i \omega T_{s}}\right)=0$ for all frequencies. Extensions to nonlinear systems with a sensitivity operator is further explored in [18]. The Bode integral constraint on $G$ is slightly more complicated:

$$
\int_{0}^{\pi} \frac{\log \left|G\left(e^{-i \omega}\right)\right|}{1-\cos \omega} d \omega=\pi\left(n+1-\frac{1}{\beta}\right),
$$

where $n$ is the time delay.

Time domain performance is typically expressed using the Root Mean Squared Error (RMSE) over $N$ samples:

$$
\operatorname{RMSE}(N)=\sqrt{\frac{1}{N} \sum_{t=0}^{N-1}\left(\gamma_{i}^{t}(t)-\gamma_{i}(t)\right)^{2}}
$$

\section{STANDARDIZED WCDMA POWER CONTROL ALGORITHMS}

Several power control algorithms are standardized by 3GPP to be used in WCDMA [19]. When not considering operation in soft handover nor in compressed mode, the default closed loop algorithm in the up- and downlink is as in the following section. In addition, some parameter-enabled alternatives are described in the two following sections.

\section{A. Fixed-Step Power Control}

The power level is increased/decreased depending wheather the measured SIR is below or above target SIR, and implemented as:

$$
\begin{array}{rc}
\text { Receiver : } & e_{i}(t)=\gamma_{i}^{t}(t)-\gamma_{i}(t) \\
& s_{i}(t)=\operatorname{sign}\left(\mathrm{e}_{\mathrm{i}}(\mathrm{t})\right) \\
\text { Transmitter : } & p_{T P C, i}(t)=\Delta_{i} s_{i}(t) \\
& p_{i}(t+1)=p_{i}(t)+p_{T P C, i}(t)
\end{array}
$$

This is the default choice both in the uplink and the downlink for dedicated channels. Performance and dynamical behavior using this algorithm is further explored in [20], [21]

\section{B. Uplink Alternatives}

This alternative algorithm is a different command decoding than above and is denoted ULAlt1. It makes it possible to emulate smaller step sizes than the minimum power control step, or to turn off uplink power control by transmitting an alternating series of TPC commands. In a 5-slot cycle $(j=$ $1, \ldots, 5)$, the power update $p_{T P C, i}(t)$ in $\left.\sqrt{13 \mathrm{c}}\right)$ is computed according to:

$$
p_{T D C, i}(t)= \begin{cases}\Delta_{i} & (j=5) \&\left(\sum_{j=1}^{5} s_{i}(j)=5\right) \\ -\Delta_{i} & (j=5) \&\left(\sum_{j=1}^{5} s_{i}(j)=-5\right) \\ 0 & \text { otherwise }\end{cases}
$$

\section{Downlink Alternatives}

There are two downlink alternatives, both aiming at reducing the risk of using excessive powers. In the first one, here denoted by DLAlt1, the control commands are repeated over three consecutive slots. The second one, denoted DLAlt2, reduces the controllers ability to follow deep fades by limiting the power raise. As with the ULAlt1, the commands are decoded differently than in Section III-A, described as an alternative to $13 \mathrm{c}$ :

$p_{T D C, i}(t)= \begin{cases}-\Delta_{i} & s_{i}(t)<0 \\ \Delta_{i} & \left(s_{i}(t)>0\right) \&\left(p_{\text {sum }, i}(t)+\Delta_{i}<\delta_{\text {sum }}\right) \\ 0 & \text { otherwise }\end{cases}$

where $p_{\text {sum,i }}(t)$ is the sum of the previous $N$ power updates and $N$ and $\delta_{\text {sum }}$ are configurable parameters.

\section{Fundamental Limitations}

Some fundamental limitations for general feedback control systems will be further explored in this section, partly with references to the discussion in Section III-D. The results are exemplified using the integrating controller in (2), and the WCDMA algorithms described in Section III. The sample rate of the power control is $1500 \mathrm{~Hz}$ as in WCDMA.

\section{A. Limited Update Rate}

Consider the ideal integrating controller in (2) with $\beta=1$ and two mobiles with velocities $2 \mathrm{~m} / \mathrm{s}$ and $9 \mathrm{~m} / \mathrm{s}$. The power gain is described in Section II-B, which yields that the disturbance energy for the two mobiles is concentrated to up to $160 \mathrm{~Hz}$ and $720 \mathrm{~Hz}$ respectively. Both these frequencies are below the Nyquist freqency $(750 \mathrm{~Hz})$ and can thus be represented without alias. However, as seen in Fig. 3 a,b) only the disturbance of the first mobile is compensated for. The answer lies in the sensitivity function in Fig. 3 k, where we note that only frequencies up to $\approx 200 \mathrm{~Hz}$ are suppressed.

\section{B. Time Delays}

Time delays affect stability as with any feedback controlled system, and therefore more careful control actions have to be imposed [17]. But time delays do not only affect stability. As seen in (11), the closed-loop performance is more restricted with longer time delays, and this put restrictions on the sensitivity as well, see Fig. 3k). We also conclude that, in this case with a linear controller, not much can be gained by using time delay compensation (TDC) [13]. The reduced sensitivity to disturbances is already evident when compensating for the slow mobile $(v=2 \mathrm{~m} / \mathrm{s})$ as seen in Fig. $3 \mathrm{~d}$.

\section{Limited Feedback Bandwidth}

To implement a power control algorithm such as the integrating controller in 2, information about the error $e_{i}(t)$ has to be fed back. The feedback communnication is a cost in itself, and must be restricted. We will consider it as a limited feedback bandwidth, which in the WCDMA example is 1500 bps. To optimize the update rate, one bit per sample interval can be used, and a possible coding of the control command is to feed back the sign of the error. Note that this results in the FSPC algorithm in (13). For feedback error robustness, one such command can be coded on three consecitive identical bits, resulting in the algorithm DLAlt1. This results in 

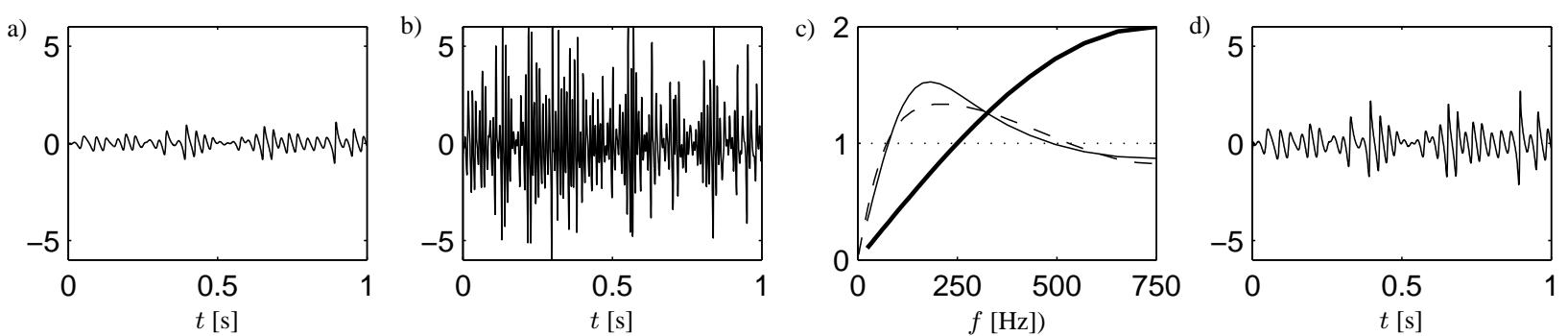

Fig. 3. Performance of the integrating controller for $\beta=1$ and no delay (a-c) and $\beta=0.3$ and one slot delay (c-d) in terms of RMSE in (12). a) $e_{i}(t)$, slow mobile ( $\mathrm{v}=2 \mathrm{~m} / \mathrm{s}), \operatorname{RMSE}=0.29, \mathrm{~b}) e_{i}(t)$, fast mobile $\left.(\mathrm{v}=20 \mathrm{~m} / \mathrm{s}), \mathrm{RMSE}=1.8, \mathrm{c}\right)\left|S\left(e^{i \omega T_{s}}\right)\right|$ of the ideal controller (thick), with delay and $\beta=0.3$ (thin) and the latter with TDC (dashed) d) $e_{i}(t)$, slow mobile, RMSE $=0.60$.

a)

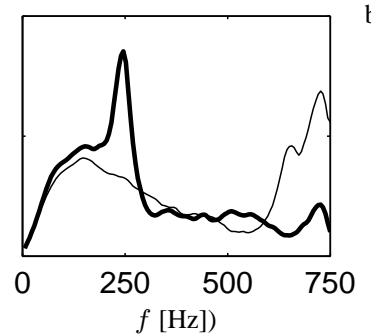

b)

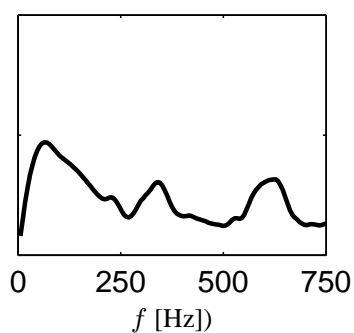

c)

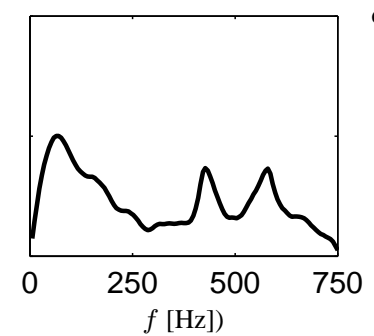

d)

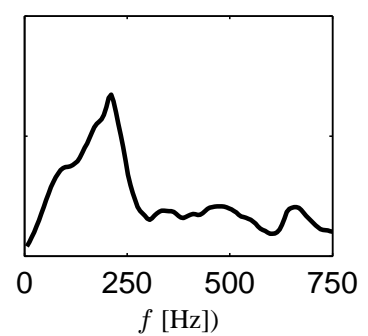

Fig. 4. Sensitivity function of a) FSPC algorithm (thick), FSPC with TDC (thin), b) ULAlt1, c) DLAlt1, d) DLAlt2
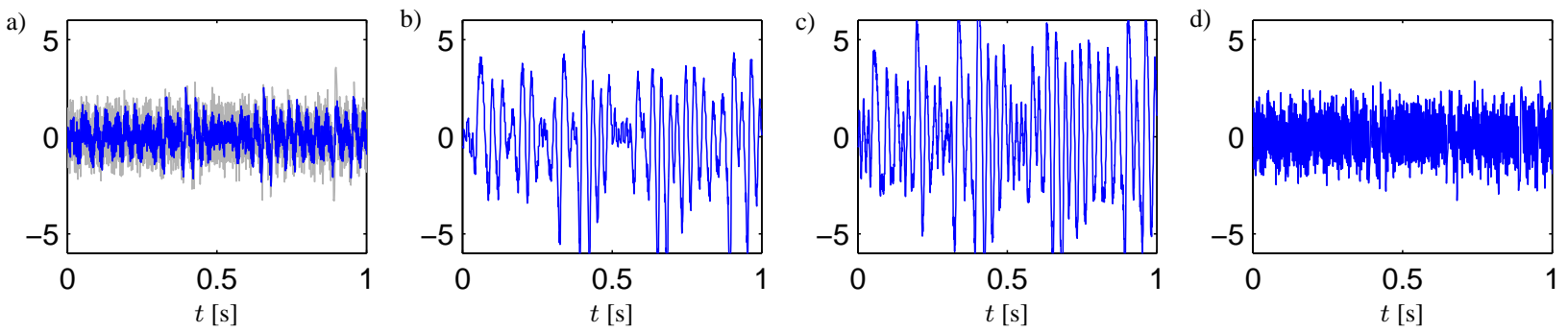

Fig. 5. Disturbance rejection when tracking a slow mobile $(v=2 \mathrm{~m} / \mathrm{s})$ a) FSPC algorithm (gray), RMSE=1.1, FSPC with TDC (black), RMSE $=0.81, \mathrm{~b}$ ) ULAlt1, RMSE=2.3, c) DLAlt1, RMSE=3.0, d) DLAlt2, RMSE=1.8.

a power update rate of $500 \mathrm{~Hz}$. Clearly, there is a trade-off between the accuracy and robustness of representing the error $e_{i}(t)$ and the one hand, and update rate on the other. Fig. 4 provides sensitivity function estimates as in (9) corresponding to the algorithms described in Section III. Resonance peaks are present more or less in all of the sensitivity functions. This is due to the nonlinearity (the sign function) together with the dynamics, which result in an oscillative behavior. The FSPC oscillation period $T_{\text {osc }}$ can directly be related to the time delay $n$ as $T_{o s c}=4 n+2$ as predicted in [21]. This corresponds to the resonance peak in Fig. 4 a. The effect of TDC is that this resonance is shifted upwards in frequency, and thus not as crucial. As expected, the algorithms using more than one bit per command yield worse disturbance rejection. This is also seen in the time domain plots in Fig. 5illustrating compensating for the slow mobile.

\section{Measurement Errors}

When no measurement noise is present, an intuitive design objective that stems from $(7)$ is $S(q)=0$ and $G(q)=1$. This would result in perfect disturbance rejection and perfect tracking. However, even if this would have been possible (it is not according to the Bode integral in $(10)$ ), it would not be interesting anyway when subject to noise. According to (7) this is equivalent to being maximally sensitive to measurement noise. Therefore, it is vital to consider measurement noise in the design and apply measurement filtering if necessary.

Measuring is not an instantaneous procedure, even though the measurements often are considered as samples of a continuous process. This is a relevant approximation in most power control cases. However, some related issues are brought up here.

In WCDMA, measurements are obtained from the fraction $\delta_{s}$ of the slot, which in turn corresponds to $T_{s}=1 / 1500 \mathrm{~s}$. Typical values [22] of $\delta_{s}$ include $\delta_{s}=0.1$ (considering only the four pilot symbols out of 40 symbols) and $\delta_{s}=0.25$ 
(considering the ten first symbols). These values depend on the data rate and channel configuration assigned to the user. A comparison of the filtering effects when considering a full slot average compared to a fractional slot average is found in Fig. 6 Aliasing is avoided if the frequency components over the Nyquist frequency are filtered out. This is almost the case when using the local average of the full slot (or measurement period). Conversely, aliasing effects are most likely when adopting local average over fractional slots.

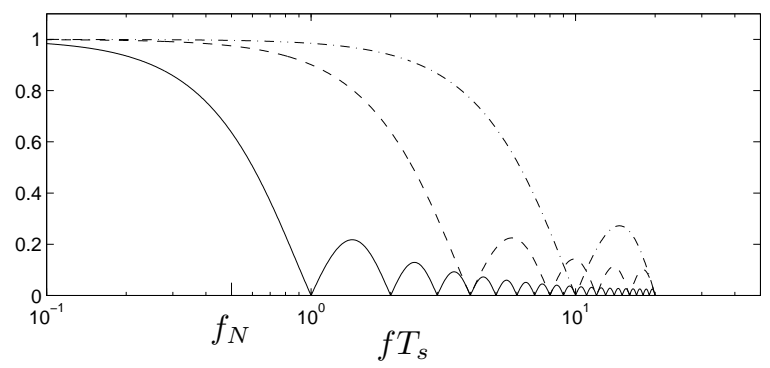

Fig. 6. Filtering effect with respect to normalized frequency of a local average filter applied to the full slot (solid) and fractional slots $\delta_{s}=0.25$ (dashed) and $\delta_{s}=0.1$ (dash-dotted). The normalization is with respect to the frequency after down-sampling, which in this case is 40 times smaller.

\section{E. Feedback Errors}

In order to keep the feedback bandwidth to a minimum, the power control commands are not code-protected to a large extent. Therefore, command errors of $0-10 \%$ are not unrealistic. This in turn affect the performance. Since the downlink power likely will be a limiting resource, it is important to minimize the risk of using to high a power. Therefore, the error protection is more crucial in the downlink. An approach more robust against single errors than FSPC is the DLAlt1, using three bits to code one command. If the command bit error probability is $p$, then this algorithm will feature a command error probability of $p^{2}(3-2 p)<<p$.

\section{F. Feasibility and Global Stability}

A necessary condition for proper operation of these algorithms is that it is possible to assign transmitter powers so that every user meets his requirements. If propagation information about all connections is be available at one point, the feasibility could be computed [6]. However, this is not plausible in practice. Implementationally tractable algorithms and load definitions is further discussed in [23].

This far only isolated connections have been in focus. The cross-connections do interact via induced interference, and this limits the possible local control actions. Convergence results for FSPC are provided in [5], [6]. In case of log-linear controllers, global stability can be expressed as the local requirement:

$$
\left|G\left(e^{i \omega}\right)\right| \leq 1
$$

which is a sufficient condition together with local loop stability [3].

\section{Conclusions}

Distributed power control algorithms can be seen as interacting local control loops. As such, a number of fundamental limitations can be derived using control theory. Thereby, aspects of limited update rates, time delays, limited feedback bandwidth, measurement errors, feedback errors and feasibility are discussed. The ability of the controller to reject disturbances is instructively described in the frequency domain by the sensitivity function. This in turn can be related to the frequency content of the disturbance. Using a log-linear model, the power gain can be seen as an additive disturbance. With a power gain describtion in the spatial frequency domain, the discussion can be made for a general mobile velocity. In light of these limitations, some central 3GPP proposals for WCDMA power control are analyzed.

\section{REFERENCES}

[1] Z. Rosberg and J. Zander. Toward a framework for power control in cellular systems. Wireless Networks, 4(3), 1998.

[2] S. Hanly and D.-N. Tse. Power control and capacity of spread spectrum wireless networks. Automatica, 35(12), 1999.

[3] F. Gunnarsson Power Control in Cellular Radio System: Analysis, Design and Estimation $\mathrm{PhD}$ thesis, Linköpings universitet, Linköping, Sweden, April 2000.

[4] S.A. Grandhi, R. Vijayan, D.J. Goodman, and J. Zander. Centralized power control in cellular radio systems. IEEE Transactions on Vehicular Technology, 42(4), 1993.

[5] J.D. Herdtner and E.K.P. Chong. Analysis of a class of distributed asynchronous power control algorithms for cellular wireless systems. IEEE Journal on Selected Areas in Communications, 18(3), Mar 2000.

[6] F. Gunnarsson and F. Gustafsson. Convergence of some power control algorithms with time delay compensation. Submitted to IEEE Transactions on Wireless Communications, 2001.

[7] G.J. Foschini and Z. Miljanic. A simple distributed autonomus power control algorithm and its convergence. IEEE Transactions on Vehicular Technology, 42(4), 1993.

[8] S.A. Grandhi, J. Zander, and R. Yates. Constrained power control. Wire less Personal Communications, 2(1), 1995.

[9] A. Salmasi and S. Gilhousen. On the system design aspects of code division multiple access (CDMA) applied to digital cellular and personal communications networks. In Proc. IEEE Vehicular Technology Conference, New York, NY, USA, May 1991.

[10] R.D. Yates. A framework for uplink power control in cellular radio systems. IEEE Journal on Selected Areas in Communications, 13(7) September 1995.

[11] M. Almgren, H. Andersson, and K. Wallstedt. Power control in a cellular system. In Proc. IEEE Vehicular Technology Conference, Stockholm, Sweden, June 1994.

[12] S. Ulukus and R. Yates. Stochastic power control for cellular radio systems. IEEE Transactions on Communications, 46(6), 1998.

[13] F. Gunnarsson and F. Gustafsson Time delay compensation in power controlled cellular radio systems IEEE Communications Letters, 5(7), Jul 2001 .

[14] F. Gunnarsson, F. Gustafsson, and J. Blom Pole placement design of power control algorithms In Proc. IEEE Vehicular Technology Conference, Houston, TX, USA, May 1999.

[15] K. Åström and B. Wittenmark. Computer Controlled Systems - Theory and Design. Prentice-Hall, Englewood Cliffs, NJ, USA, third edition, 1997.

[16] L. Ljung System Identification - Theory for the User Prentice Hall, Englewood Clifts, NJ, USA., second edition, 1999.

[17] F. Gunnarsson, J. Blom, and F. Gustafsson Power control in cellular systems subject to constraints and time delays In Proc. IEEE Global Telecommunications Conference, Sydney, Australia, November 1998.

[18] M. M. Seron, J. H. Braslavsky, and G. C. Goodwin. Fundamental Limitations in Filtering and Control. Springer-Verlag, Berlin, Germany, 1997.

[19] Release 1999 3GPP Technical Specification Group Radio Access Network. Physical radio procedures. Technical report 3G TS 25.214, 2001.

[20] L. Song, N.B. Mandayam, and Z. Gajic. Analysis of an up/down power control algorithm for the CDMA reverse link under fading. Submitted to IEEE Journal on Selected Areas in Communications, Wireless Com munications Series, 2000.

[21] F. Gunnarsson, F. Gustafsson, and J. Blom Dynamical effects of time delays and time delay compensation in power controlled DS-CDMA IEEE Journal on Selected Areas in Communications, 19(1), Jan 2001.

[22] F. Adachi, M. Sawahashi, and H. Suda. Wideband DS-CDMA for nextgeneration mobile communications systems. IEEE Communications Magazine, 36(9), 1998.

[23] E. Geijer-Lundin and F. Gunnarsson Admission control based on relative load estimates In Proc. Nordic Radio Symposium, Nynäshamn, Sweden, April 2001. 